\title{
ABBV-176, a PRLR antibody drug conjugate with a potent DNA-damaging PBD cytotoxin and enhanced activity with PARP inhibition
}

Mark G. Anderson ${ }^{1 *}$ (D), Qian Zhang ${ }^{1}$, Luis E. Rodriguez ${ }^{1}$, Claudie M. Hecquet ${ }^{1}$, Cherrie K. Donawho², Peter J. Ansell ${ }^{1}$ and Edward B. Reilly ${ }^{1}$

\begin{abstract}
Background: Prolactin receptor (PRLR) is an attractive antibody therapeutic target with expression across a broad population of breast cancers. Antibody efficacy, however, may be limited to subtypes with either PRLR overexpression and/or those where estradiol no longer functions as a mitogen and are, therefore, reliant on PRLR signaling for growth. In contrast a potent PRLR antibody-drug conjugate (ADC) may provide improved therapeutic outcomes extending beyond either PRLR overexpressing or estradiol-insensitive breast cancer populations.

Methods: We derived a novel ADC targeting PRLR, ABBV-176, that delivers a pyrrolobenzodiazepine (PBD) dimer cytotoxin, an emerging class of warheads with enhanced potency and broader anticancer activity than the clinically validated auristatin or maytansine derivatives. This agent was tested in vitro and in vivo cell lines and patient derived xenograft models.

Results: In both in vitro and in vivo assays, ABBV-176 exhibits potent cytotoxicity against multiple cell line and patient-derived xenograft breast tumor models, including triple negative and low PRLR expressing models insensitive to monomethyl auristatin (MMAE) based PRLR ADCs. ABBV-176, which cross links DNA and causes DNA breaks by virtue of its PBD warhead, also demonstrates enhanced anti-tumor activity in several breast cancer models when combined with a poly-ADP ribose polymerase (PARP) inhibitor, a potentiator of DNA damage.

Conclusions: Collectively the efficacy and safety profile of ABBV-176 suggest it may be an effective therapy across a broad range of breast cancers and other cancer types where PRLR is expressed with the potential to combine with other therapeutics including PARP inhibitors.
\end{abstract}

Keywords: Prolactin receptor PRLR, Antibody drug conjugate, Pyrrolobenzodiazepine dimer, Combination therapy, Poly (ADP-ribose) polymerase I

\footnotetext{
* Correspondence: mark.g.anderson@abbvie.com

${ }^{1}$ AbbVie Inc., Oncology Discovery, 1 North Waukegan Rd., North Chicago, IL 60064-6099, USA
}

Full list of author information is available at the end of the article

C C The Author(s). 2021 Open Access This article is licensed under a Creative Commons Attribution 4.0 International License, which permits use, sharing, adaptation, distribution and reproduction in any medium or format, as long as you give appropriate credit to the original author(s) and the source, provide a link to the Creative Commons licence, and indicate if changes were made. The images or other third party material in this article are included in the article's Creative Commons licence, unless indicated otherwise in a credit line to the material. If material is not included in the article's Creative Commons licence and your intended use is not permitted by statutory regulation or exceeds the permitted use, you will need to obtain permission directly from the copyright holder. To view a copy of this licence, visit http://creativecommons.org/licenses/by/4.0/ The Creative Commons Public Domain Dedication waiver (http://creativecommons.org/publicdomain/zero/1.0/) applies to the data made available in this article, unless otherwise stated in a credit line to the data. 


\section{Background}

Worldwide, over 2.1 million women are impacted by breast cancer each year, causing the most cancer-related deaths among women. In the U.S., over 275,000 women are expected to be diagnosed with invasive breast cancer this year, and over 1 in 8 women will develop the disease over her lifetime, making it the second most diagnosed cancer next to skin cancer for women [1, 2]. Although development of targeted agents has led to improved outcomes in patients with breast cancer, $50 \%$ of patients with advanced disease treated with current standard of care will suffer from disease progression [3]. This observation highlights the need for additional treatment options for patients with advanced breast cancer including Her2+, TDM-1 refractory breast cancer and triple negative breast cancer (TNBC). Evolving evidence suggests that therapies targeting the prolactin receptor (PRLR) may provide a distinct advantage in treating these patient populations [4-6].

PRLR is a type 1 cytokine receptor implicated in the initiation and progression of breast cancer [7-10]. The PRLR/PRL (prolactin ligand) signaling axis contributes to estrogen-insensitive breast cancer growth and development. PRLR is expressed in many human breast tumors where its levels are elevated compared to normal breast tissue $[11,12]$. High circulating levels of its ligand, PRL, correlate with increased risk for developing breast cancer [13] and with disease progression and reduced response to tamoxifen [13-16]. PRLR has been shown to confer resistance against chemotherapeutic agents including docetaxel, doxorubicin and cisplatin [17].

The functional attributes and tumor expression profile of PRLR make it an attractive target for therapeutic intervention in breast cancer and potentially other malignancies. For example, the neutralizing antibody LFA102 antagonizes PRLR signaling and proliferation in breast cancer cells and regresses PRL-dependent tumor xenografts [10]. As a large percentage of breast tumors express estrogen receptors and remain sensitive to estrogen, an unconjugated PRLR antibody would be anticipated to be effective primarily only in those breast tumor subtypes where estradiol no longer functions as a mitogen and are, therefore, reliant on PRLR signaling for growth. While LFA-102 was well tolerated as an unconjugated antibody in Phase 1 clinical trials in patients with metastatic breast and prostate cancer, efficacy was limited [18].

In contrast, an antibody drug conjugate (ADCs) targeting PRLR may provide superior therapeutic outcomes including and extending beyond estradiol-insensitive breast cancer populations since its mechanism of action is not dependent on hormonal dependency. Elevated expression of PRLR in tumor versus normal breast tissue may enable an acceptable therapeutic window for PRLR
ADCs. An anti-PRLR ADC comprised of a high-affinity function blocking anti-PRLR IgG1 antibody (REGN2878) conjugated to the cytotoxic maytansine derived DM1, has shown significant anti-tumor activity against several breast cancer xenograft tumor models although the activity was limited to those models with high levels of PRLR expression [4].

Herein we describe the characteristics and functional attributes of ABBV-176, a novel pyrrolobenzodiazepine (PBD) ADC targeting PRLR across multiple breast cancer models including low PRLR models and models insensitive to MMAE (monomethyl auristatin)-based PRLR ADCs. Ultimately, the full clinical potential of the PRLR ADC may be realized by combinations with other therapeutics that complement the PBD MoA. Consequently, studies combining PRLR-PBD ADCs with other therapeutics including potentiators of DNA damaging agents were also evaluated.

\section{Methods \\ Antibodies and reagents}

A panel of PRLR-specific mAbs was generated using standard hybridoma technology following immunization with the extracellular domain (ECD, 25-234) of PRLR. Candidates were screened based on binding properties, and epitope, and candidates were humanized as IgG1 isotypes before conjugation to MMAE [19]. The lead mAb, h16f, was identified based on improved affinity, epitope binding properties and activity, and then engineered to include an S238C point mutation (S239C based on Kabat numbering system [20]] to permit sitespecific DAR 2 conjugation to a PBD dimer (SGD-1882) via mc-Val-Ala linker as previously described [21].

Recombinant human, cynomolgus, and murine PRLR extracellular domains with a His-tag (huPRLR $25-234$, cyPRLR $_{25-234}$ and muPRLR $20-229$ ) were expressed in and purified from HEK293 cells. Recombinant ECD of rat PRLR (residues 20-229 fused with poly-His tag at C-terminal), was purchased from Sino Biological Inc. and further purified by gel filtration using Superdex200 (GE Healthcare) in 10 mM HEPES, pH 7.4, 150 mM NaCl, 3 mM EDTA.

\section{Surface plasmon resonance: human and cynomolgus PRLR extracellular domain binding assay}

Binding kinetics of ABBV-176 and the h16f parental mAb to recombinant human and cynomolgus PRLR ECD were determined by surface plasmon resonance-based measurements made on Biacore T200 instrument (GE Healthcare) using previously described methods [22].

\section{Cell culture}

Human tumor cell lines from ATCC were expanded in culture upon receipt and cryopreserved to provide cells at a similar stage passage for all subsequent experiments. 
For cell lines not authenticated in the 6 months before use, their PRLR expression levels were confirmed by FACS analysis. T47D and 22RV1 were maintained in RPMI-1640 (Gibco Invitrogen, \#11875) supplemented with $10 \%$ fetal bovine serum (FBS). Cell lines CAMA1, AN3CA BT-474, MCF7, MDA-MB-361, SKBR3, UACC812, MDA-MB-231, SMOV2 [23], SW403, HuH7, HepG2 and HEK-293 were cultured in DMEM (Gibco-Invitrogen, \#11965) supplemented with 10\% FBS. All cell culture was done at $37^{\circ} \mathrm{C}$ in a humidified incubator with $5 \% \mathrm{CO}_{2}$. All non-transformed cells were cultured using manufacturer-recommended conditions: NHBE, HUVEC, HMEC, PrEC, and NHDF were obtained from LONZA; primary normal HUF, HRE, MCF10A, HMVEC, and THLE-3 cells were obtained from ATCC; and HRMC, were obtained from iXCells Biotechnologies. All assays were performed within two passages after thawing.

\section{Retroviral infection}

Retroviral infections were performed using the Lenti-X viral system (Clontech) according to manufacturer's instructions, with PRLR sequences from human and cynomolgus monkey cloned into the pLVX-IRES-puro vector.

\section{Binding ELISA and fluorescence-activated cell sorting (FACS) analysis}

ELISA binding assays with PRLR ECD were performed as previously described, except $0.25 \mu \mathrm{g} / \mathrm{ml}$ of ECD protein was bound to plates [24].

For cellular binding studies, cells were harvested and sorted as previously described [24] using anti-PRLR (mouse version of h16f) or control antibody $((-) \mathrm{Co})$. When necessary to dissociate cell aggregates, cells were briefly treated with Accutase (Millipore, \#SCR005). Data was analyzed using Becton Dickinson FACSDIVA software. For quantitative determination of PRLR on the cell surface, QIFIKIT (Dako) was carried out according to the manufacturer's instructions, as previously described [25].

\section{Cytotoxicity assay}

Cell lines were plated at 500-5000 cells/well for CellTiter-Glo assays as previously described [25], including a huIgG1-PBD as a negative control ADC.

\section{Western blot comparison for PRLR expression}

Two to three replicate untreated tumors were subjected to Western blot analysis as previously described [24] with anti-PRLR (inv359200, Invitrogen) and anti-actin (Sigma, \# A5441). To assess relative PRLR expression levels for xenograft lysates, the low PRLR-expressing MCF7 xenograft ( $~ 8000$ receptors/cell in culture) lysates were run in parallel as an internal standard. For each individual MCF7 lysate, the total PRLR signal was divided by the actin signal and the average value then used to normalize the other CDX and PDX lysates relative to the MCF7 PRLR value.

\section{In vivo studies}

Female SCID (Huh-7 LOT, $n=9$ per dose group and HepG2, $n=10$ per dose group) and SCID Beige (BT-474, $n=10$ per dose group) mice were obtained from Charles River Laboratories (Wilmington, MA) and handled and studies carried out as previously described [25] with the following exceptions. For BT-474.FP2 (BT-474 sub-line) and HepG2, $5 \times 10^{6}$ viable cells were inoculated subcutaneously (s.c.), and for HUH-7 LOT (liver orthotopic subline), $1 \times 10^{6}$ for cells were similarly inoculated. Animals were tagged and followed individually throughout the experiment and dose groups were caged together. Randomization by the matched distribution algorithm and tumor volume calculations, two times weekly, used Study Director version 3.1.399 (Studylog Systems, Inc., South San Francisco). Mice were euthanized by isofluorane inhalation with no oxygen mixture to anesthetize the animals until they became unconscious and stopped breathing in compliance with AbbVie's Institutional Animal Care and Use Committee guidelines, when tumor volume was $\geq 1500 \mathrm{~mm}^{3}$ or skin ulcerations occurred.

The PDX $N=3$ study (with single animals, in individual HEPA ventilated cages) was conducted using similar methodologies at Champions Oncology (Baltimore, MD) in compliance with Champion's Institutional Animal Care and Use Committee and the National Institutes of Health Guide for Care and Use of Laboratory Animals guidelines in a facility accredited by the Association for the Assessment and Accreditation of Laboratory Animal Care [26]. Investigators at Champions were blinded to the identity of the dosing materials.

The expanded CTG-0869 $(n=10)$ and CTG-0670 $(n=$ 8) studies were conducted similarly with the following modifications. PDX tumors grown in SCID Bg female were harvested when they reached a size of approximately $1000 \mathrm{~mm}^{3}$ size and finely chopped into a homogenous brie. PDX brie $(0.2 \mathrm{ml}$ of a $1: 1$ mixture of brie and Matrigel (BD Biosciences)) was inoculated s.c. into the lower right flank of recipient SCID Bg female study animals. Mice were treated intraperitoneal with the indicated doses of ADCs, and in combination studies with $200 \mathrm{mkd}$ of veliparib administered orally b.i.d for 21 days. Veliparib, synthesized at Abbott Laboratories (Abbott Park, IL), was formulated in $0.9 \%$ saline.

\section{Statistical analysis}

$\mathrm{IC}_{50}$ and $\mathrm{EC}_{50}$ values were determined by nonlinear regression analysis of concentration response curves using GraphPad Prism 6.0 (GraphPad Software, La Jolla, CA). 
For tumor studies, mean tumor volume and standard error shown were analyzed using the Student's t-test for differences in $\mathrm{T} / \mathrm{C}$ values (StatView, SAS Institute, Cary, $\mathrm{NC}$ ). For the PDX $N=3$ screening study, the selectivity and anti-tumor activity efficacy of ABBV-176 was by the Welch t-test correlating the log transformed relative tumor volume at the time of maximum tumor growth inhibition (TGI), and mixed effect modeling, comparing the log of fold change in tumor volume (versus the first time point) for every available time point using $\mathrm{R}$ software (Bell Laboratories, Madison, WI). Both tests were used, with $\geq 1$ significant test describing treatment efficacy. Survival curves were generated and analyzed using the JMP statistical software v7 (SAS institute, Cary, NC). The Log-Rank (Mantle-Cox) test was used to calculate the survival improvement of treatments.

\section{Results}

\section{Generation of a PRLR ADC}

Candidate antibodies were initially conjugated to MMAE payloads and evaluated for their ability to inhibit the growth of the $\mathrm{BT}-474$, an $\mathrm{ER}+$, progesterone receptor (PR)-, HER2+ breast cancer cell line. BT-474 has $\sim 10$,
000 PRLR receptors per cell, which is lower than the number typically necessary to mediate effective ADC killing, suggesting that efficient internalization may be a critical component for activity of a PRLR ADC (Table 1). This tumor cell line may, therefore, also serve as a surrogate measure of ADC internalization properties. Based on cytotoxic assay results with BT-474, the h16f maleimide-conjugated mc-Val-Cit-MMAE DAR4 ADC was identified as the lead candidate with the most potent inhibitory activity. An antibody with an engineered cysteine (C239) was generated to permit site-specific malemide conjugation of the PBD dimer with DAR 2 and the final ADC conjugate was designated as ABBV-176.

\section{Binding properties of ABBV-176 for PRLR}

The binding of both parental antibody h16f and ABBV176 were similar to both human and cynomolgus monkey recombinant PRLR extracellular domain (25-234), with high apparent affinity $\left(\mathrm{EC}_{50}\right.$ approximately 20 and 18 pM, Fig. 1A and B) and the absence of appreciable binding to mouse or rat PRLR ECD $\left(\mathrm{EC}_{50}>67 \mathrm{nM}\right)$ by ELISA. As measured by Biacore analysis, the affinities of h16f and ABBV-176 to the recombinant form of the

Table 1 PRLR Expression and ABBV-176 Cytotoxicity in Human Tumor Cell Lines

\begin{tabular}{|c|c|c|c|}
\hline & PRLR Expression $^{\mathrm{a}}$ & ABBV-176 IC $50(n M)^{b}$ & h16f-MMAE IC $_{50}(\mathrm{nM})^{\mathrm{b}}$ \\
\hline \multicolumn{4}{|l|}{ Breast cancer } \\
\hline T47D & 26,000 & $0.0055(0.006)$ & 0.22 \\
\hline CAMA1 & 10,000 & 0.01 & 5.2 \\
\hline BT-474 & 10,000 & $0.24(0.04)$ & 0.56 \\
\hline MCF7 & 8000 & $0.32(0.3)$ & $>22$ \\
\hline MDA-MB-361 & $5-10,000$ & $0.77(0.2)$ & 0.96 \\
\hline SKBR3 & $5-10,000$ & 0.26 & 3.67 \\
\hline UACC812 & $\sim 3500$ & $>22$ & $>22$ \\
\hline MDA-MB-231 & Below detection & $>22$ & $>22$ \\
\hline \multicolumn{4}{|l|}{ Prostate } \\
\hline $22 \mathrm{RV} 1$ & 8000 & 0.01 & $>22$ \\
\hline \multicolumn{4}{|l|}{ Endometrial } \\
\hline AN3CA & 8300 & 0.6 & 22 \\
\hline \multicolumn{4}{|l|}{ Ovarian } \\
\hline SMOV2 & $\sim 2300$ & 0.16 & $>22$ \\
\hline \multicolumn{4}{|l|}{ Colorectal } \\
\hline SW403 & 11,000 & 0.11 & 17 \\
\hline \multicolumn{4}{|l|}{ Liver } \\
\hline HepG2 & n.d. & 8.6 & $>22$ \\
\hline $\mathrm{HuH}-7$ & $\sim 14,000$ & 5.2 & $>22$ \\
\hline
\end{tabular}

$I C_{50}$ half maximal inhibitory concentration, PRLR prolactin receptor, $n . d$. not done due to cell aggregation

${ }^{a}$ Cell surface PRLR per cell is indicated based on quantitative FACS

${ }^{b}$ Cell viability was determined following incubation with indicated ADC for $144 \mathrm{~h}$. The values represent IC ${ }_{50}$ s. Averages are shown when multiple experiments were performed, with standard deviations in parentheses. Unconjugated anti-PRLR antibody does not inhibit growth of any of these cell lines 
A

$$
\text { huPRLR }_{(25-234)} \text { ELISA Binding }
$$

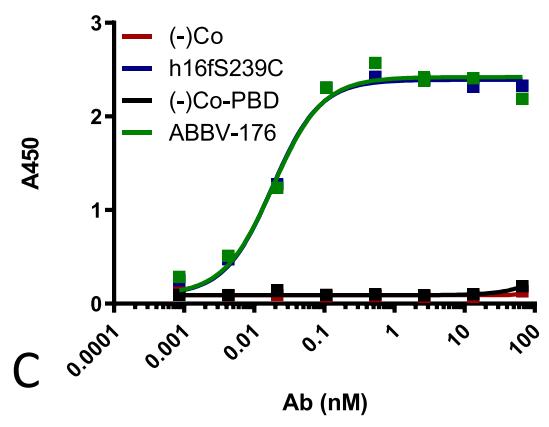

T47D FACS Binding

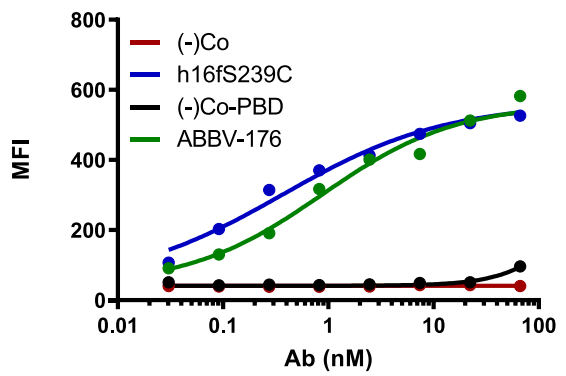

$\mathrm{E}$

HEK-293 huPRLR FACS Binding

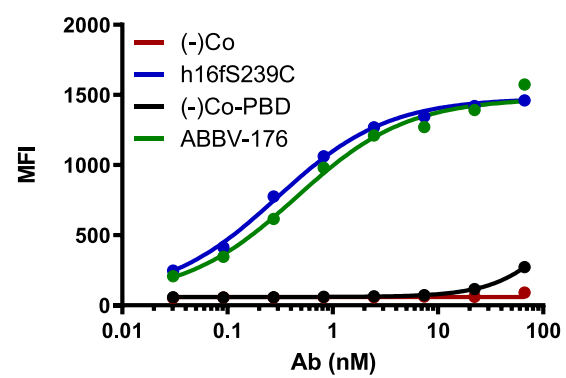

B cyPRLR $_{(25-234)}$ ELISA Binding

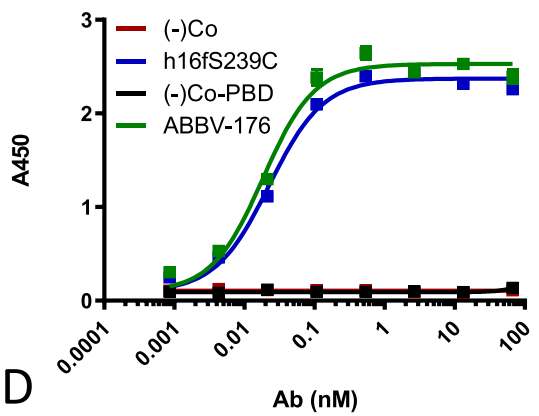

MCF7 FACS Binding

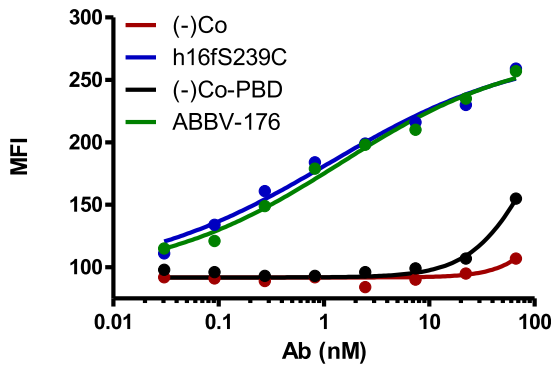

$\mathrm{F}$

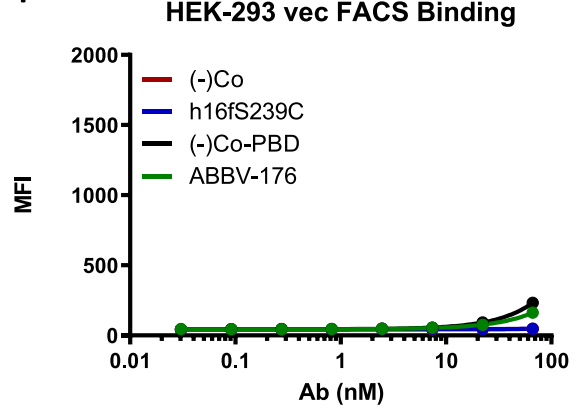

Fig. 1 ABBV-176 Binding to PRLR. Binding to immobilized PRLR extracellular domain recombinant protein by ELISA is shown in for ABBV-176 and its unconjugated antibody, along with control antibody (unconjugated and PBD-conjugated) to human (A), cynomolgus (B) proteins ECDs. Binding to cells expressing PRLR was assessed by fluorescence activated cell sorting (FACS) analysis of the PRLR-high cell line T47D (C) and the PRLR low cell line MCF-7 (D) with titration curves for geomeans plots shown. Specificity of binding of the anti-PRLR antibody and ABBV-176 is shown in HEK-293 cells engineered to over express PRLR (E) and not in negative control HEK-293 cells (F)

human PRLR ECD were comparable (KD of $1.0 \mathrm{nM}$ ), and similar to cynomolgus PRLR ECD (0.7 nM).

Binding of h16f and ABBV-176 to cell surface PRLR was measured by fluorescence activated cell sorting (FACS). Both h16f and ABBV-176 bind comparably to cells expressing human wild-type PRLR including a high expressing tumor cell line (T47D Fig. 1C), a low expressing tumor cell line (MCF-7, Fig. 1D) and HEK-293 cells engineered to express human PRLR (Fig. 1E). No significant cell surface binding was observed with the control antibody or its PBD conjugate (Fig. 1C-E), and neither h16f nor ABBV-176 appreciably bound to control HEK293 vector control cells (Fig. 1F).
In vitro potency of ABBV-176 against tumor cell lines and correlation with PRLR expression

h16f-MMAE and ABBV-176 conjugates were evaluated for their ability to inhibit the growth of a panel of 25 breast cancer cell lines expressing different levels of PRLR (Table 1). Measurement of PRLR receptor densities on these tumor cells permitted a preliminary assessment of the correlation between receptor expression and sensitivity to ADC-mediated killing. Results indicated cell line sensitivity to killing by the ADC correlated with PRLR mRNA and protein expression. PRLR was overexpressed in both HER2+ and HER2- tumors. Every tumor cell line sensitive to killing by h16f-MMAE ADC was 
equally or more sensitive to killing by ABBV-176. Additionally, several tumor cell lines with lower levels of PRLR were sensitive to killing by the PBD conjugate but were largely insensitive to h16f-MMAE. These results suggest that ABBV-176 (h16f (S239C)-PBD) with a payload more potent than h16f-MMAE, has the potential to expand the breadth of sensitive tumors.

A panel of normal cell lines representing kidney, breast, liver, lung, prostate, and vascular endothelium, (HUVEC, HUF, HRE, HMEC, NHBE, PrEC, HMVEC, THLE-3 and MCF-10A) was tested for presence of PRLR, and minimal PRLR protein was observed by Western blot. Consistent with this result, these cells were largely unaffected by treatment with ABBV-176, similar to the non-targeting control ADC, and had much less cell killing than free PBD cytotoxin (Supplemental data).

Since the PRLR/PRL axis has been implicated in other tissues, including reproductive tissues and prostate $[9,27-$ 31], h16f-MMAE and ABBV-176 conjugates were evaluated for their ability to inhibit the growth of non-breastderived tumor cell lines (Table 1). Although the levels of
PRLR RNA in these cell lines was generally much lower than observed for many breast cancer cell lines, several cell lines including ovarian, endometrial, prostate and colorectal cell lines were sensitive to killing by ABBV-176, but not h16f-MMAE. These results suggest that the activity of ABBV-176 may extend to other PRLR-expressing tumor indications beyond breast cancer.

\section{ABV-176 in vivo efficacy in PRLR-expressing tumor models}

The in vivo efficacy of ABBV-176 was evaluated in the BT-474 FP2 human xenograft breast tumor model. BT474 is a low PRLR-expressing tumor cell line with $\sim 10$, 000 receptors per cell (Table 1). ABBV-176 at a single dose of $0.5 \mathrm{mg} / \mathrm{kg}$ was effective $(p \leq 0.05)$ in regressing established tumors, while h16f-MMAE was also efficacious $(p \leq 0.05)$ in this model, although at a much higher dose of $3 \mathrm{mg} / \mathrm{kg}$ (Fig. 2A). Notably, these and all other in vivo dosing regimens of ABBV-176 in these studies had no significant impact on body weight of the mice compared to controls over the course of the study

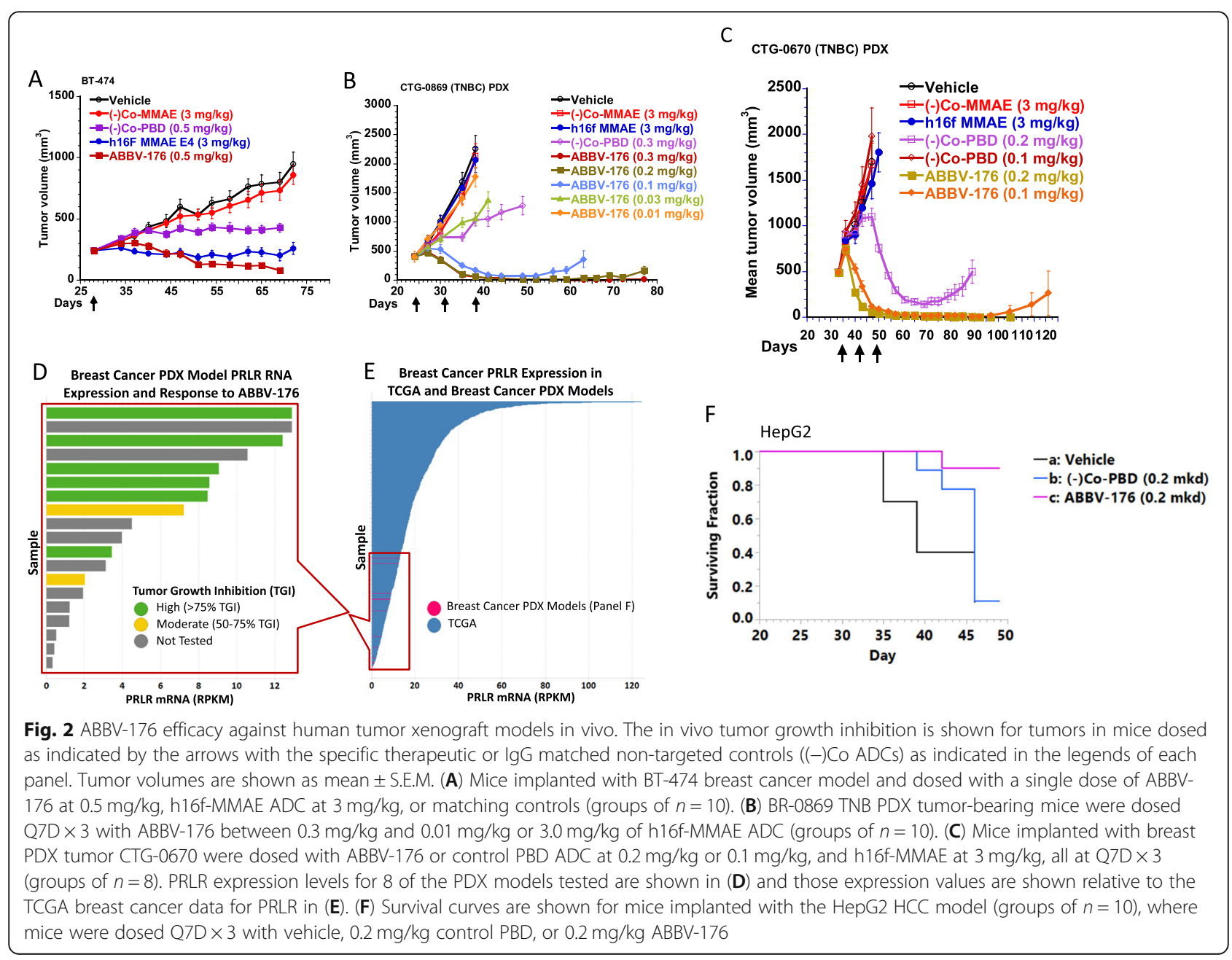


(unpublished data), nor were there any gross physiologic changes observed to indicate an impact on normal tissues. The non-targeting PBD dimer ADC control (-)CoPBD exhibited variable activity in the in vivo models, likely driven by high potency of the ADC and tumor related enhanced permeability and retention effects, consistent with previous findings $(22$ and references therein).

In a patient-derived xenograft (PDX) study with CTG-0869 (0.8x the total PRLR as in the MCF7 cell line), ABBV-176 also exhibited potent anti-tumor activity when compared to vehicle and non-targeted control PBD ADC at the highest dose, $0.5 \mathrm{mg} / \mathrm{kg}$, as well as the $0.3 \mathrm{mg} / \mathrm{kg}, 0.2 \mathrm{mg} / \mathrm{kg}$, and $0.1 \mathrm{mg} / \mathrm{kg}$ doses administered Q7D $\times 3$, which induced durable tumor regression, with similar results seen for CTG-0670 (Fig. 2B and C, $(p \leq 0.05)$, and Table 2). At $0.1 \mathrm{mg} /$ $\mathrm{kg}$, tumors started to regrow by day 63 , whereas tumors treated with $0.03 \mathrm{mg} / \mathrm{kg}$ had a minimal tumor growth delay. These results are in sharp contrast to the MMAE ADC, which had no impact on this model (Fig. 2B solid blue circles).

As PDX models have not been grown in cell culture and have not been highly passaged in mice, they are likely to be more closely related to patient tumors. The anti-tumor activity of ABBV-176 was therefore evaluated in an expanded set of primarily, but not exclusively, TNB PDX tumor models that express a range from weak to moderate PRLR. To determine the relative amount of PRLR present in these PDX models, two approaches were used. By Western blot analysis, total PRLR protein levels ranges from $0.15 \mathrm{x}$ to $3.83 \mathrm{x}$ the total PRLR levels in MCF7, as indicated in the Table 2. Consistent with the total protein levels, when the RNA seq data was compared to TCGA breast cancer data, most of these models expressed low levels of PRLR relative to patient samples, implying that these models express PRLR equal to, or lower than, most breast cancer $(\mathrm{BrCa})$ patient tumors (Fig. 2D-E). Significantly, ABBV-176 was efficacious in 10 of the 12 models tested. To evaluate these responses in more depth, a single dose experiment was performed with CTG-0670. Single dose studies with CTG-0670 demonstrated high potency at $0.1 \mathrm{mg} / \mathrm{kg}$ $(p \leq 0.05)$, confirming the efficacy of ABBV-176 in $\mathrm{BrCa}$ (Fig. 3A). Consistent with the results from the in vitro assay (Table 1), these data indicate that ABBV-176 is a more potent $\mathrm{ADC}$ conjugate than auristatin-based ADCs and its activity can extend to lower PRLR-expressing tumors.

To determine the in vivo activity outside of $\mathrm{BrCa}$, the hepatocellular carcinoma line HepG2 was tested for sensitivity to ABBV-176. Despite low PRLR expression

Table 2 Human Breast Cancer Models ABBV-176; N= 3 Screen Study

\begin{tabular}{|c|c|c|c|c|}
\hline Model ID & PRLR Density vs MCF7 & Drug Doses $^{a}$ & ABBV-176 Efficacy ${ }^{b}$ & ER/PR/HER2 BRCA Status \\
\hline CTG-1124 & 0.15 & 0.2 & High (84\%) & $\begin{array}{l}\text { ER+/PR+ } \\
\text { BRCA } 1 \text { def }\end{array}$ \\
\hline CTG-0012 & 0.75 & 0.5 & High (90\%) & $\begin{array}{l}\text { TNBC } \\
\text { BRCA } 1 \text { def }\end{array}$ \\
\hline CTG-0869 & 0.8 & 0.5 & High (93\%) & $\begin{array}{l}\text { TNBC } \\
\text { BRCA } 1 \text { mut }\end{array}$ \\
\hline CTG-0670 & 1.64 & 0.5 & High (91\%) & $\begin{array}{l}\text { TNBC } \\
\text { BRCA } 1 \text { def, BRCA } 2 \text { mut }\end{array}$ \\
\hline CTG-0033 ${ }^{\mathrm{C}}$ & 2.06 & 0.2 & High (79\%) & $\begin{array}{l}\text { HER2+ } \\
\text { BRCA n.d. }\end{array}$ \\
\hline CTG-1171 & 0.42 & 0.2 & High (82\%) & $\begin{array}{l}\text { TNBC } \\
\text { BRCA 1, } 2 \text { mut }\end{array}$ \\
\hline CTG-1019 & 0.37 & 0.2 & High (83\%) & $\begin{array}{l}\text { TNBC } \\
\text { BRCA 1, } 2 \text { mut }\end{array}$ \\
\hline CTG-1242 & 3.83 & 0.2 & High (83\%) & $\begin{array}{l}\text { TNBC } \\
\text { BRCA n.d. }\end{array}$ \\
\hline CTG-0052 & 0.46 & 0.2 & Moderate (59\%) & $\begin{array}{l}\text { TNBC } \\
\text { BRCA wild type }\end{array}$ \\
\hline CTG-0017 & 0.27 & 0.2 & Moderate (62\%) & $\begin{array}{l}\text { TNBC } \\
\text { BRCA 1, } 2 \text { mut }\end{array}$ \\
\hline CTG-1520 & 0.56 & 0.5 & Low (48\%) & $\begin{array}{l}\text { TNBC } \\
\text { BRCA wild type }\end{array}$ \\
\hline
\end{tabular}

BRCA breast cancer DNA associated repair gene, def deficient, ER estrogen receptor, HER2 human epidermal growth factor receptor $2, I P$ intraperitoneal, mut mutation, n.d. not determined, $P R$ progesterone receptor, $T G /$ tumor growth inhibition, $T N B C$ triple negative breast cancer

${ }^{a}$ Doses were administered IP Q7D $\times 3$, at the dose level indicated in $\mathrm{mg} / \mathrm{kg}$

${ }^{\mathrm{b}}$ Low $=25-50 \% \mathrm{TGl}$, Moderate $=50-75 \%$ TGl, High $>75 \%$ TGI compared to control PBD ADC treated group $(p<0.0001)$

${ }^{\mathrm{C}}$ This study was performed with larger size treatment groups $(N=6)$ 
A CTG-0670 (TNBC) PDX

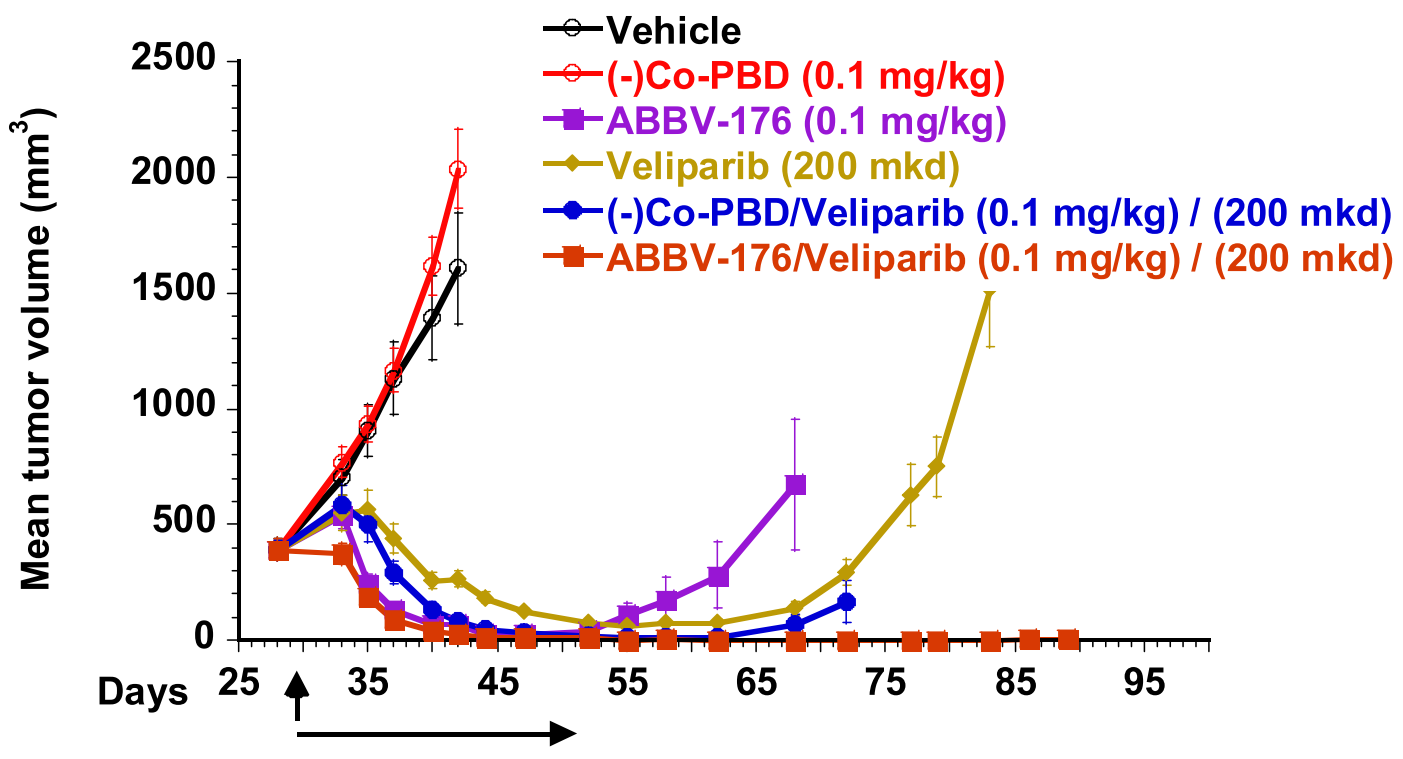

B Huh-7 LOT

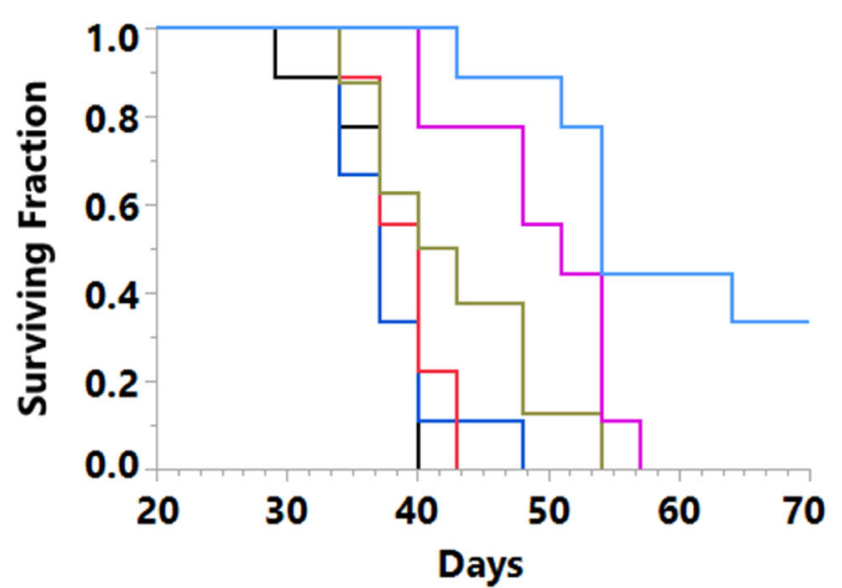

-Vehicle

- (-)Co-PBD (0.2 mkd)

- Veliparib (200 mkd)

- (-)Co-PBD (0.2 mkd)/Neliparib (200 mkd)

-ABBV-176 (0.2 mkd)

-ABBV-176 (0.2 mkd)/Veliparib (200 mkd)

Fig. 3 ABBV-176 is active in combination in vivo with the PARP inhibitor Veliparib. Mice with CTG-0670 breast cancer PDX tumors were treated with a single dose of $0.1 \mathrm{mg} / \mathrm{kg} \mathrm{ABBV}-176,0.1(-)$ Co-PBD $\mathrm{mg} / \mathrm{kg}$, vehicle, or Veliparib BID $\times 21$ days, or a combination of each ADC with Veliparib at the same amount and schedule (groups of $n=10)(\mathbf{A})$. Mice with HuH-7 LOT HCC tumors were treated Q7D $\times 3$ with either 0.2 mg/kg ABBV176, $0.2(-)$ Co-PBD mg/kg, vehicle, or Veliparib BID $\times 21$ days, or a combination of one ADC with Veliparib at the same amount and schedule (groups of $n=9$ ). (B)

(0.09x total PRLR as MCF7), HepG2 had tumor growth delay ( $p<0.001$ compared to vehicle, $p<0.005$ compared to $\mathrm{Co}-\mathrm{PBD}$ ) when treated with ABBV-176 at $0.2 \mathrm{mg} / \mathrm{kg}$ Q7D $\times 3$ as shown in the survival chart (Fig. 2F). Activity was also observed in the hepatocellular carcinoma line Huh-7 LOT (see combination studies described below, and shown in Fig. 3B).
ABBV-176 combination with PARP inhibition

Maximizing the full clinical potential of ADCs will likely require combinations with other therapeutics. Accordingly, based on complementary mechanisms of action, the effects of combining ABBV-176 with the PARP inhibitor ABT-888 (Veliparib) were investigated in the CTG-0670 TNB BRCA1 deficient, BRCA2 mutant PDX 
tumor models. In the CTG-0670, one dose at $0.1 \mathrm{mg} / \mathrm{kg}$ of ABBV-176 monotherapy treatment was active so the number of CRs was used to compare monotherapy and combination treatment activity shown in Fig. 3A. Combination treatment resulted in increased number of CRs - 8 CRs following combination treatment versus no CRs following monotherapy. Significant tumor growth inhibition was observed through day 89 , whereas tumor regrowth was observed following either treatment alone, or treatment with the Veliparib and PBD control combination (Fig. 3A).

To investigate the combinational activity beyond breast cancer models, the activity of ABBV-176 and Veliparib was evaluated in two HCC models, HepG2 and HuH-7 LOT, that express low levels of PRLR (0.09x and 0.26x total PRLR levels in MCF7, respectively). ABBV-176 impacted survival of mice bearing HepG2 tumors, but no significant effect of combination with veliparib was observed (Fig. $2 \mathrm{~F}$ and data not shown). In contrast, in mice implanted with the higher PRLR expressing $\mathrm{HuH}-7$ tumors LOT, the combination treatment of veliparib and ABBV-176 increased survival $(p<$ $0.05)$ compared to ABBV-176 or $(p<0.0001)$ compared to veliparib alone (Fig. 3B).

\section{Discussion}

New modalities of therapeutic intervention are needed for breast cancer patients, especially for the triple negative subtype, as well as estrogen-independent and relapsed metastatic breast cancers. PRLR is a receptor whose expression is enhanced in breast cancers and presents a potential target for delivering cytotoxic payloads independent of ER, PR or HER2 dependence/expression. Previously described PRLR-targeting therapies include a mutated PRLR ligand, G129L, [32], a neutralizing antiPRLR antibody, LFA-102, a HER2-PRLR bispecific/ADC approach [5], and a microtubule inhibitor-based ADC, REGN2878-DM1 [4]. In contrast, ABBV-176 is a PRLRtargeting ADC that leverages both the rapid internalization properties of PRLR [5], and a highly potent PBD cytotoxin. Based on results from mouse xenograft tumor models, these properties translate into a highly active therapeutic with activity extending to those tumors with low PRLR expression - as few as 10,000 receptors per cell - which is notably below levels typically present on tumor cells targeted by ADCs. Significantly, ABBV-176 demonstrated anti-tumor efficacy against low PRLR expressing $\mathrm{BrCa}$ PDX models. RNA expression levels in these $\mathrm{BrCa}$ sensitive PDX tumors corresponded to the lower third of all BrCA tumors within the TCGA database suggesting that the majority of $\mathrm{BrCa}$ tumors may express sufficient levels of PRLR to render them sensitive to ABBV-176.

Multiple PRLR positive breast tumor cell lines including triple negative and low PRLR expressers are also sensitive to ABBV-176. The efficacy of ABBV-176 was superior compared to the h16F- MMAE ADC with potent activity observed in PDX models including some that were completely refractory to h16-MMAE at tenfold higher doses. In vitro and in vivo anti-tumor activity was also seen with ABBV-176 in hepatocellular carcinoma cell lines refractory to the MMAE ADC. Although PRLR expression has generally been primarily associated with breast cancers, additional evaluation of the efficacy of ABBV-176 in non-breast cancer indications that express low levels of PRLR may be warranted.

A key challenge with therapeutic ADCs is the potential for on-target and off-target toxicities. In the case of ABBV-176, these concerns are exacerbated by the potent PBD payload that necessitates lower therapeutic doses in patients and subsequently reduced exposures as shown in [33]. This in turn may limit ADC availability to the tumor site especially if normal tissue disposition is observed. Ultimately combination approaches may be needed to minimize the dose levels needed of any single agent to minimize each individual therapy's toxicities, while maximizing clinical benefit.

The PBD cytotoxin cross links DNA and causes DNA breaks, requiring DNA repair mechanisms for cell survival. Poly ADP ribose polymerase (PARP1) binds to DNA breaks and is critical for base excision repair and nucleotide excision repair pathways, and PARP inhibitors are approved for treatment of $\mathrm{BrCa}$ patients with germline BRCA mutations [34]. This mechanism of action suggests that PARP inhibitors such as Veliparib may enhance the activity of PBD-dimer ADCs. The combination of ABBV-176 and the PARP inhibitor Veliparib demonstrated enhanced activity in the BRCA1 deficient triple negative breast model CTG-0670 as well as the liver CDX HuH-7 LOT model, but not the HepG2 liver model. Due to a mutation in the FANCC gene, HuH-7 cells have impaired FANCD2 nuclear foci-formation in response to irradiation, while HepG2 cells do not. After DNA damage, FANCD2 and FANCI are activated by monoubiquitination to complex with other Franconi anemia proteins, BRCA2, RAD51, and aditional DNA damage repair proteins, critical for repair of interstrand crosslinks. The damage caused by the PBD delivered by ABBV-176, coupled with this DNA repair impairment, could drive the PARP inhibition combination effects in the absence of BRCA-deletions [35, 36]. Recently, Zhong et al. has also reported improved antitumor effects of a 5 T4-PBD ADC when combined with the PARP inhibitor Oliparib [37]. ABBV-176 therefore offers an expanded breadth of efficacy, potentially even beyond breast cancer, while combination with potentiators of DNA damage offers opportunities to reduce dose levels and toxicities and potentially enhance efficacy in patients. 


\section{Conclusions}

ABBV-176 is a potential therapeutic for metastatic breast cancer patients that have lost sensitivity to ERtargeting modalities and as well those that relapse after HER2-based approaches such as Herceptin, Kadcyla patients. ABBV-176 binds to PRLR, is rapidly internalized, and delivers a potent PBD cytotoxin to tumor cells. This DNA crosslinking payload offers a distinct mechanism of action from $\mathrm{BrCa}$ standard of cares that include antiestrogens, microtubule inhibitors, CDK4/6 inhibitors, and HER2 targeting therapies, that combines with PARP inhibition and can contribute to circumventing resistance to these treatments.

\begin{abstract}
Abbreviations
PRLR: Prolactin receptor; ADC: Antibody-drug conjugate; MMAE: Monomethyl auristatin; PBD: Pyrrolobenzodiazepine; PARP: Poly-ADP ribose polymerase; TNBC: Triple negative breast cancer; HER2: Human epidermal growth factor receptor 2; PRL: Prolactin; ECD: Extracellular domain; FACS: Fluorescence activated cell sorting; ELISA: Enzyme enzyme-linked immunosorbent assay; (-)Co: Non targeted human IgG; PDX: Patient-derived xenograft; mkd: Milligrams per kilogram per day; Q7D: Once every 7 days dosing; BrCa: Breast cancer; HCC: Hepatocellular carcinoma; IP: Intraperitoneal; mut: Mutation; n.d.: Not determined; ER: Estrogen receptor; PR: Progesterone receptor; TGI: Tumor growth inhibition; BID: Twice daily
\end{abstract}

\section{Supplementary Information}

The online version contains supplementary material available at https://doi. org/10.1186/s12885-021-08403-5.

Additional file 1: Fig. S1. Western blot analysis for PRLR in normal/ nontransformed human cells. Panel A: HUVEC (umbilical vein endothelium), PrEC (prostate), HUF (primary uterine fibroblasts), NHBE (bronchial epithelium), HMEC (mammary epithelium), HRE (primary renal mixed epithelium), HRMC (renal mesangial cells), BT474 (breast cancer) as a positive control. Panel B: MCF10A (normal breast line), HMVEC (microvascular endothelium), and THLE-3 (immortalized liver). Examples of In vitro cell killing assay. Panel C: HMEC cells. Panel D: HRMC cells.

Additional file 2: Fig. S2. Full Western blots from S1. Panels A and B: M are markers, $\mathrm{X}$ are irrelevant cell lines, and remaining lanes as in S1.

\section{Acknowledgements}

The authors thank H. Doug Falls of AbbVie for assistance with FACS of PRLRexpressing cell lines.

\section{Authors' contributions}

MGA: conception and framework design of the studies, scientific guidance during the project, and initial draft of the manuscript; QZ: performing binding, Western blot, and proliferation assays; LER: performing and anlayzing in vivo studies xeograft; $\mathrm{CMH}$ : guidance and coordination of analysis of in vivo studies with patient-derived xenografts; CKD: design, oversight and analysis of all in vivo studies; PJA: expression analysis with PDX models and tumors; and ERB: conception, scientific guidance during the project, and co-writing of the manuscript. All authors contributed to the review and editing of the manuscript. All authors read and approved the final manuscript.

\section{Funding}

The design, study conduct, and financial support for this research were provided by AbbVie. AbbVie participated in the interpretation of data, review, and approval of the publication.

\section{Availability of data and materials}

All data generated or analysed during this study are included in this published article and its supplementary information files.

\section{Declarations}

\section{Ethics approval and consent to participate}

These studies did not involve human participants.

Animal studies followed AbbVie's Institutional Animal Care and Use Committee and the NIH Guide for Care and Use of Laboratory Animals Guidelines in a facility accredited by the Association for the Assessment and Accreditation of Laboratory Animal Care. Animal Studies at Champions followed Champion's Institutional Animal Care and Use Committee and the National Institutes of Health Guide for Care and Use of Laboratory Animals guidelines in a facility accredited by the Association for the Assessment and Accreditation of Laboratory Animal Care.

\section{Consent for publication}

Not Applicable.

\section{Competing interests}

MGA, QZ, LER, CMH, PJA, and EBR are employees of AbbVie. CKD was an employee of AbbVie at the time of the study.

\section{Author details}

${ }^{1}$ AbbVie Inc., Oncology Discovery, 1 North Waukegan Rd., North Chicago, IL 60064-6099, USA. ²Formerly AbbVie, Oncology Discovery, 1 North Waukegan Rd., North Chicago, IL 60064, USA.

Received: 21 November 2020 Accepted: 25 May 2021

Published online: 09 June 2021

\section{References}

1. Breast cancer [https://www.who.int/cancer/prevention/diagnosis-screening/ breast-cancer/en/].

2. U.S. Breast Cancer Statistics [https://www.breastcancer.org/symptoms/ understand_bc/statistics].

3. Martei YM, Matro JM. Identifying patients at high risk of breast cancer recurrence: strategies to improve patient outcomes. Breast Cancer (Dove Med Press). 2015;7:337-43. https://doi.org/10.2147/BCTT.S91981.

4. Kelly MP, Hickey C, Makonnen S, Coetzee S, Jalal S, Wang Y, et al. Preclinical activity of the novel anti-prolactin receptor (PRLR) antibody-drug conjugate REGN2878-DM1 in PRLR-positive breast cancers. Mol Cancer Ther. 2017; 16(7):1299-311. https://doi.org/10.1158/1535-7163.MCT-16-0839.

5. Andreev J, Thambi N, Perez Bay AE, Delfino F, Martin J, Kelly MP, et al. Bispecific antibodies and antibody-drug conjugates (ADCs) bridging HER2 and prolactin receptor improve efficacy of HER2 ADCs. Mol Cancer Ther. 2017;16(4):681-93. https://doi.org/10.1158/1535-7163.MCT-16-0658.

6. Zhou $Y$, Zong $H$, Han L, Xie $Y$, Jiang $H$, Gilly J, et al. A novel bispecific antibody targeting CD3 and prolactin receptor (PRLR) against PRLRexpression breast cancer. J Exp Clin Cancer Res. 2020;39(1):87. https://doi. org/10.1186/s13046-020-01564-4.

7. Oakes SR, Robertson FG, Kench JG, Gardiner-Garden M, Wand MP, Green JE, et al. Loss of mammary epithelial prolactin receptor delays tumor formation by reducing cell proliferation in low-grade preinvasive lesions. Oncogene. 2007;26(4):543-53. https://doi.org/10.1038/sj.onc.1209838.

8. Arendt LM, Rugowski DE, Grafwallner-Huseth TA, Garcia-Barchino MJ, Rui H, Schuler LA. Prolactin-induced mouse mammary carcinomas model estrogen resistant luminal breast cancer. Breast Cancer Res. 2011;13(1):R11. https://doi. org/10.1186/bcr2819.

9. Damiano JS, Wasserman E. Molecular pathways: blockade of the PRLR signaling pathway as a novel antihormonal approach for the treatment of breast and prostate cancer. Clin Cancer Res. 2013;19(7):1644-50. https://doi. org/10.1158/1078-0432.CCR-12-0138.

10. Damiano JS, Rendahl KG, Karim C, Embry MG, Ghoddusi M, Holash J, et al. Neutralization of prolactin receptor function by monoclonal antibody LFA102, a novel potential therapeutic for the treatment of breast cancer Mol Cancer Ther. 2013;12(3):295-305. https://doi.org/10.1158/1535-7163. MCT-12-0886.

11. Touraine P, Martini JF, Zafrani B, Durand JC, Labaille F, Malet C, et al. Increased expression of prolactin receptor gene assessed by quantitative polymerase chain reaction in human breast tumors versus normal breast tissues. J Clin Endocrinol Metab. 1998;83(2):667-74. https://doi.org/10.1210/ jcem.83.2.4564. 
12. Faupel-Badger JM, Duggan MA, Sherman ME, Garcia-Closas M, Yang XR, Lissowska J, et al. Prolactin receptor expression and breast cancer: relationships with tumor characteristics among pre- and post-menopausal women in a population-based case-control study from Poland. Horm Cancer. 2014;5(1):42-50. https://doi.org/10.1007/s12672-013-0165-7.

13. Tworoger SS, Eliassen AH, Sluss P, Hankinson SE. A prospective study of plasma prolactin concentrations and risk of premenopausal and postmenopausal breast cancer. J Clin Oncol. 2007;25(12):1482-8. https://doi. org/10.1200/JCO.2006.07.6356.

14. Holtkamp W, Nagel GA, Wander HE, Rauschecker HF, von Heyden D. Hyperprolactinemia is an indicator of progressive disease and poor prognosis in advanced breast cancer. Int J Cancer. 1984;34(3):323-8. https:// doi.org/10.1002/ijc.2910340307.

15. Bhatavdekar JM, Patel DD, Karelia NH, Shah NG, Ghosh N, Vora HH, et al. Can plasma prolactin predict tamoxifen resistance in patients with advanced breast cancer? Eur J Surg Oncol. 1994;20(2):118-21.

16. Bhatavdekar JM, Patel DD, Shah NG, Vora HH, Suthar TP, Ghosh N, et al. Prolactin as a local growth promoter in patients with breast cancer: GCRI experience. Eur J Surg Oncol. 2000;26(6):540-7. https://doi.org/10.1053/ ejso.2000.0943.

17. LaPensee EW, Schwemberger SJ, LaPensee CR, Bahassi el M, Afton SE, BenJonathan N: Prolactin confers resistance against cisplatin in breast cancer cells by activating glutathione-S-transferase. Carcinogenesis 2009, 30(8): 1298-1304, DOl: https://doi.org/10.1093/carcin/bgp120.

18. Agarwal N, Machiels JP, Suarez C, Lewis N, Higgins M, Wisinski K, et al. Phase I study of the prolactin receptor antagonist LFA102 in metastatic breast and castration-resistant prostate Cancer. Oncologist. 2016;21(5):535-6. https:// doi.org/10.1634/theoncologist.2015-0502.

19. Doronina SO, Toki BE, Torgov MY, Mendelsohn BA, Cerveny CG, Chace DF, et al. Development of potent monoclonal antibody auristatin conjugates for cancer therapy. Nat Biotechnol. 2003;21(7):778-84. https://doi.org/10.1038/ nbt832.

20. Kabat AM. Sequences of proteins of immunological interest. 5th ed. Bethesda, MD: U.S. Department of Health and Human Services; 1991.

21. Jeffrey SC, Burke PJ, Lyon RP, Meyer DW, Sussman D, Anderson M, et al. A potent anti-CD70 antibody-drug conjugate combining a dimeric pyrrolobenzodiazepine drug with site-specific conjugation technology. Bioconjug Chem. 2013;24(7):1256-63. https://doi.org/10.1 021/bc400217g.

22. Anderson MG, Falls HD, Mitten MJ, Oleksijew A, Vaidya KS, Boghaert ER, et al. Targeting Multiple EGFR Expressing Tumors with a Highly Potent Tumor-Selective Antibody Drug Conjugate. Mol Cancer Ther. 2020;19(10): 2117-25. https://doi.org/10.1158/1535-7163.MCT-20-0149.

23. Yonamine K, Hayashi K, lida T. Establishment and characterization of human ovarian clear cell adenocarcinoma cell line (SMOV-2), and its cytotoxity by anticancer agents. Hum Cell. 1999;12(3):139-48.

24. Wang J, Goetsch L, Tucker L, Zhang Q, Gonzalez A, Vaidya KS, et al. Anti-cmet monoclonal antibody ABT-700 breaks oncogene addiction in tumors with MET amplification. BMC Cancer. 2016;16(1):105. https://doi.org/10.1186/ s12885-016-2138-z.

25. Wang J, Anderson MG, Oleksijew A, Vaidya KS, Boghaert ER, Tucker $L$, et al. ABBV-399, a c-met antibody-drug conjugate that targets both METamplified and c-met-overexpressing tumors, irrespective of MET pathway dependence. Clin Cancer Res. 2017;23(4):992-1000. https://doi.org/10.1158/1 078-0432.CCR-16-1568.

26. Bharathy N, Berlow NE, Wang E, Abraham J, Settelmeyer TP, Hooper JE, et al. The HDAC3-SMARCA4-miR-27a axis promotes expression of the PAX3: FOXO1 fusion oncogene in rhabdomyosarcoma. Sci Signal. 2018;11(557).

27. Ahonen TJ, Xie J, LeBaron MJ, Zhu J, Nurmi M, Alanen K, et al. Inhibition of transcription factor Stat5 induces cell death of human prostate cancer cells. J Biol Chem. 2003;278(29):27287-92. https://doi.org/10.1074/jbc.M304307200.

28. Otte JM, Otte C, Beckedorf S, Schmitz F, Vonderhaar BK, Folsch UR, et al. Expression of functional prolactin and its receptor in human colorectal cancer. Int J Color Dis. 2003;18(1):86-94. https://doi.org/10.1007/s00384-002-0414-7.

29. Robertson FG, Harris J, Naylor MJ, Oakes SR, Kindblom J, Dillner K, et al. Prostate development and carcinogenesis in prolactin receptor knockout mice. Endocrinology. 2003;144(7):3196-205. https://doi.org/10.1210/en.2003-0068.

30. Goffin V, Hoang DT, Bogorad RL, Nevalainen MT. Prolactin regulation of the prostate gland: a female player in a male game. Nat Rev Urol. 2011;8(11): 597-607. https://doi.org/10.1038/nrurol.2011.143.
31. Yeh Y-T, Lee K-T, Tsai C-J, Chen Y-J, Wang S-N. Prolactin promotes hepatocellular carcinoma through Janus kinase 2. World J Surg. 2012;36(5): 1128-35. https://doi.org/10.1007/s00268-012-1505-4.

32. Scotti ML, Langenheim JF, Tomblyn S, Springs AE, Chen WY. Additive effects of a prolactin receptor antagonist, G129R, and herceptin on inhibition of HER2-overexpressing breast cancer cells. Breast Cancer Res Treat. 2008; 111(2):241-50. https://doi.org/10.1007/s10549-007-9789-z.

33. Lemech C, Woodward N, Chan N, Mortimer J, Naumovski L, Nuthalapati S, et al. A first-in-human, phase 1, dose-escalation study of ABBV-176, an antibody-drug conjugate targeting the prolactin receptor, in patients with advanced solid tumors. Investig New Drugs. 2020;38(6):1815-25. https://doi. org/10.1007/s10637-020-00960-Z.

34. Morales J, Li L, Fattah FJ, Dong Y, Bey EA, Patel M, et al. Review of poly (ADP-ribose) polymerase (PARP) mechanisms of action and rationale for targeting in cancer and other diseases. Crit Rev Eukaryot Gene Expr. 2014; 24(1):15-28. https://doi.org/10.1615/CritRevEukaryotGeneExpr.2013006875.

35. Nalepa G, Clapp DW. Fanconi anaemia and cancer: an intricate relationship. Nat Rev Cancer. 2018;18(3):168-85. https://doi.org/10.1038/nrc.2017.116.

36. Palagyi A, Neveling K, Plinninger U, Ziesch A, Targosz BS, Denk GU, et al. Genetic inactivation of the Fanconi anemia gene FANCC identified in the hepatocellular carcinoma cell line $\mathrm{HuH}-7$ confers sensitivity towards DNAinterstrand crosslinking agents. Mol Cancer. 2010;9(1):127. https://doi.org/1 0.1186/1476-4598-9-127.

37. Zhong H, Chen C, Tammali R, Breen S, Zhang J, Fazenbaker C, et al. Improved therapeutic window in BRCA-mutant tumors with antibody-linked Pyrrolobenzodiazepine dimers with and without PARP inhibition. Mol Cancer Ther. 2019;18(1):89-99. https://doi.org/10.1158/1535-7163.MCT-18-0314.

\section{Publisher's Note}

Springer Nature remains neutral with regard to jurisdictional claims in published maps and institutional affiliations.
Ready to submit your research? Choose BMC and benefit from:
- fast, convenient online submission
- thorough peer review by experienced researchers in your field
- rapid publication on acceptance
- support for research data, including large and complex data types
- gold Open Access which fosters wider collaboration and increased citations
- maximum visibility for your research: over $100 \mathrm{M}$ website views per year
At BMC, research is always in progress. 